\title{
Correlation Functions of the One-Dimensional Bose Gas in the Repulsive Case
}

\author{
V. E. Korepin
}

Leningrad Department of the V. A. Steklov Mathematical Institute, Fontanka, 27, SU-191011, Leningrad, USSR

\begin{abstract}
The one dimensional Bose gas is considered in the repulsive case. The ground state of the system is the Dirac sea with a finite density. The correlation function of the currents is presented in the form of the series, the $n^{\text {th }}$ term being the contribution of $n$ vacuum particles. In the strong coupling limit $c \rightarrow \infty$ the $n^{\text {th }}$ term decreases as $c^{-n}$. In the weak coupling limit $c \rightarrow 0$ the series is also essentially simplified. The decomposition gives the uniform approximation in the distance between the currents. The arguments in favour of convergence of the series are given.
\end{abstract}

\section{Introduction}

In the present paper we consider the one dimensional Bose gas, which is equivalent to the quantum non-linear Schrödinger(NS) equation. We use the abbreviation NS to denote the model. Its Hamiltonian is equal to

$$
\mathbf{H}=\int_{0}^{L} d x\left(\partial_{x} \psi^{+} \partial_{x} \psi+c \psi^{+} \psi^{+} \psi \psi-h \psi^{+} \psi\right),\left[\psi(x), \psi^{+}(y)\right]=\delta(x-y) .
$$

We use the approach of paper [1], where the problem was imbedded in the quantum inverse scattering method (QISM) [2-4]. The notations and definitions of paper [1] are exploited also. The formulae of this paper are cited as (number of the formula) [1]. The NS model was studied in the thermodynamic limit in [5-7], where the ground state (the physical vacuum) was constructed. The system of transcendental equations (s.t.e.) (1.5) [1] for this state looks like:

$$
\varphi_{j}=2 \pi j+\pi N, \varphi_{j}=\lambda_{j} L+\sum_{\substack{k=-N / 2 \\ k \neq j}}^{N / 2} \Phi\left(\lambda_{j}-\lambda_{k}\right), \Phi(\lambda)=i \ln \left(\frac{\lambda+i c}{\lambda-i c}\right) .
$$

The integer number $j$ takes all the values in the interval $[-N / 2, N / 2]$. The number $(N+1)($ odd $)$ is the number of the particles in the vacuum. In the thermodynamic limit $L \rightarrow \infty, N / L=$ const momenta $\lambda_{j}$ - the solutions of the equations (1.1) fill the 
interval $[-q, q]$ with the density $\rho\left(\lambda_{k}\right)=1 / L\left(\lambda_{k+1}-\lambda_{k}\right)$. It satisfies the equation

$$
\left(1-\frac{1}{2 \pi} \mathbf{K}\right) \rho=\frac{1}{2 \pi} \text {. }
$$

The integral operator $\mathbf{K}$ acts on $\rho$ as follows

$$
(\mathbf{K} \rho)(\mu)=\int_{-q}^{q} K(\mu, \lambda) \rho(\lambda) d x ; \quad K(\lambda, \mu)=\frac{2 c}{c^{2}+(\lambda-\mu)^{2}} .
$$

It should be noted that $N / L=\int \rho(\lambda) d \lambda$. The eigenfunction of the Hamiltonian corresponding to the physical vacuum will be denoted by $|\Omega\rangle$. There are two kinds of excitations near the physical vacuum-the hole and the particle. Fermi momentum $q$ is defined by the condition that the energy of the excitations is zero on the boundary of the Fermi zone. The energy density in the physical vacuum can be written as an integral:

$$
\frac{1}{L} \sum_{j=-N / 2}^{N / 2} \varepsilon\left(\lambda_{j}\right) \rightarrow \int_{-q}^{q} \varepsilon(\lambda) \rho(\lambda) d \lambda .
$$

Here $\varepsilon(\lambda)$ is one-particle energy $\varepsilon(\lambda)=\lambda^{2}-h$.

The scattering matrix of these excitations can be calculated by the method of paper [8]. Dressed scattering phase is calculated by means of bare scattering phase $\Phi(1.1)$ and dressing equations. The scattering matrix of two holes $S_{h h}\left(\lambda_{2}, \lambda_{1}\right)=$ $\exp \left\{i F\left(\lambda_{2}, \lambda_{1}\right)\right\}, q>\lambda_{2}>\lambda_{1}>-q$ is defined by the dressing equation

$$
F\left(\lambda_{2}, \lambda_{1}\right)-\frac{1}{2 \pi} \int_{-q}^{q} K\left(\lambda_{2}, \mu\right) F\left(\mu, \lambda_{1}\right) d \mu=\Phi\left(\lambda_{2}-\lambda_{1}\right) .
$$

Function $F\left(\lambda_{2} \lambda_{1}\right)$ can be extended out of the interval $[-q, q]$ by means of this equation. It permits us to calculate the particle-hole $S$ matrix $S_{p h}\left(\lambda_{p}, \lambda_{h}\right)=$ $\exp \left\{-i F\left(\lambda_{p}, \lambda_{h}\right)\right\}, \lambda_{p}>q>\lambda_{h}>-q$. To calculate the particle-particle $S$ matrix $\left(\lambda_{2}>\lambda_{1}>q\right) S_{p p}\left(\lambda_{2}, \lambda_{1}\right)=\exp \left\{i F\left(\lambda_{2}, \lambda_{1}\right)\right\}$, one has to solve the equation for $F$ and to extend the phase $F\left(\lambda_{2}, \lambda_{1}\right)$ out of the interval.

The method of the calculation of norms of the eigenstates was developed in [9]. It will be used in the present paper. In the thermodynamic limit the square of the norm of the physical vacuum (1.6) [1] is equal to

$$
\operatorname{det}_{N}\left(\varphi^{\prime}\right)=\left[\prod_{j=1}^{N} 2 \pi L \rho\left(\lambda_{j}\right)\right] \operatorname{det}(\mathbf{1}-\mathbf{K} / 2 \pi) ; \quad \varphi_{j k}^{\prime}=\partial \varphi_{j} / \partial \lambda_{k} .
$$

The last factor in (1.5) is the determinant of the integral operator (1.2). It should be noted that the norms of the Bethe wave functions for the XXX model were calculated in the thermodynamic limit in paper [10]. The derivation of the formula (1.5) for the generalized model is given in Appendix A. F. A. Smirnov and the author noted that the ratio of the determinants det $\varphi^{\prime}$ of the excited state and the vacuum is generated by the change of the entropy.

The correlation function of the fields was studied in this model in the strong coupling limit in papers [11-15]. The long-wave asymptotics of these correlation functions can be calculated at any coupling constant [16]. 
In the present paper the correlation function of currents $j(x)=\psi^{+}(x) \psi(x)$ is calculated. In the strong coupling limit it is the elementary function:

$$
\frac{\left\langle\Omega\left|: j\left(x_{1}\right) j\left(x_{2}\right):\right| \Omega\right\rangle}{\langle\Omega \mid \Omega\rangle}=\left(\frac{q}{\pi}\right)^{2}-\left[\frac{\sin q\left(x_{1}-x_{2}\right)}{\pi\left(x_{1}-x_{2}\right)}\right]^{2} .
$$

It should be noted that the asymptotics of the correlation function at $\left|x_{1}-x_{2}\right| \rightarrow \infty$ can be elementary calculated at any $c$

$$
\frac{\left\langle\Omega\left|j\left(x_{1}\right) j\left(x_{2}\right)\right| \Omega\right\rangle}{\langle\Omega \mid \Omega\rangle} \rightarrow\left[\frac{\langle\Omega|j(0)| \Omega\rangle}{\langle\Omega \mid \Omega\rangle}\right]^{2}=\left[\int_{-q}^{q} \rho(\lambda) d \lambda\right]^{2} .
$$

We shall construct the consistent perturbation theory for the correlation function of the currents, which gives, for example, the improved version of the $1 / c$ expansion. The $n^{\text {th }}$ term of the series, constructed below, gives the contribution of $n$ particles, which are present in the physical vacuum. In this sense our approach is different from the bootstrap program [17], where the $n^{\text {th }}$ term of the series gives the contribution of $n$ excitations near the physical vacuum. In our paper the generalized model of the paper [1] is used, it is characterized by the arbitrary function $\ell(\lambda)$. By means of the method of the paper [9] we can explicitly evaluate the dependence of the correlation function on $\ell(\lambda)$.

The plan of the paper is the following. In Sect. 2 the main result of the paper is presented. The perturbation theory for the correlation function of the currents is described. In the rest of the paper the formulae of Sect. 2 are proved. In Sect. 3 the algebraic questions are considered. In the case of the finite number of the particles in the vacuum, the formulae generating correlation function by its irreducible part are derived. In Sect. 4 the dressing equations are constructed. It should be noted that in this case the dressing equations are non-linear. They are similar to the Liouville equation. In Sect. 5 all the formulae of Sect. 2 are proved. Section 6 is the conclusion.

\section{The Correlation Function of the Currents}

To construct the decomposition of the correlation function, it is useful to consider the mean value of the product of two currents with respect to $k$-particle eigenfunction of the Hamiltonian $\left\langle\Psi_{k}\left|j\left(x_{1}\right) j\left(x_{2}\right)\right| \Psi_{k}\right\rangle$. All the information about this mean value, which we need here, can be extracted from Sect. 1 of paper [1]. As this mean value depends only on $\left|x_{1}-x_{2}\right|$ it is sufficient to consider $\left\langle\Psi_{k}|j(x) j(0)| \Psi_{k}\right\rangle$, $x>0$. This mean value can be expressed in terms of $\mathbf{Q}_{1}$ - the operator of number of particles on the interval $[0, x]$ :

$$
\left\langle\Psi_{k}|j(x) j(0)| \Psi_{k}\right\rangle=\frac{1}{2} \frac{\partial^{2}}{\partial x^{2}}\left\langle\Psi_{k}\left|\mathbf{Q}_{1}^{2}\right| \Psi_{k}\right\rangle
$$

The mean value of the operator $\mathbf{Q}_{1}^{2}$ with respect to $k$-particle eigenfunction can be uniquely represented in the form of a "polynomial":

$$
\begin{aligned}
\left\langle\Psi_{k}\left|\mathbf{Q}_{1}^{2}\right| \Psi_{k}\right\rangle & =\sum_{n=0}^{k} \sum_{m=0}^{k-1} J_{n . m}^{(k)} x^{n} y^{m}, \\
\left\langle\Psi_{k} \mid \Psi_{k}\right\rangle & =\operatorname{det}_{k}\left(\varphi^{\prime}\right), \quad y=L-x .
\end{aligned}
$$


Here $L$ is the length of the box, the coefficients $J_{n \cdot m}^{(k)}$ are rational functions of $\lambda_{j}$ and $\exp \left\{i x \lambda_{j}\right\}$. The most important is $J_{00}^{(k)}$, which is called the irreducible part $I_{k}=J_{00}^{(k)}$. In the next sect. We shall see that all the coefficients $J_{n, m}^{(k)}$ in (2.2) can be expressed in terms of irreducible parts $I_{i}$, where $i \leqq k$. The properties of the irreducible part, which will be used below are as follows. It can be written in the form

$$
I_{k}\left(\left\{\lambda_{j}\right\}\right)=\sum_{\left\{\lambda_{j}\right\}=\left\{\lambda^{+}\right\} \cup\left\{\lambda^{-}\right\} \cup\left\{\lambda^{0}\right\}} e^{-i x \sum_{t=1}^{n}\left(\lambda_{t}^{+}-\lambda_{\ell}^{-}\right)} \mathscr{A}_{k}^{n}\left(\left\{\lambda^{+}\right\},\left\{\lambda^{-}\right\},\left\{\lambda^{0}\right\}\right) .
$$

Here the sum is taken over all the partitions of the set $\left\{\lambda_{j}\right\}, j=1, \ldots, k$ into three disjoint subsets $\left\{\lambda^{+}\right\},\left\{\lambda^{-}\right\},\left\{\lambda^{0}\right\}$, the number of the elements in the subsets being equal to: card $\left\{\lambda^{+}\right\}=\operatorname{card}\left\{\lambda^{-}\right\}=n$, card $\left\{\lambda^{0}\right\}=k-2 n, n \leqq[k / 2]$. The Fourier coefficients of the irreducible part $\mathscr{A}_{k}^{n}$ are rational functions of momenta $\lambda_{j}$. They are simply changed under the complex conjugation $\mathscr{A}^{*}\left(\left\{\lambda^{+}\right\},\left\{\lambda^{-}\right\},\left\{\lambda^{0}\right\}\right)=$ $\mathscr{A}\left(\left\{\lambda^{-}\right\},\left\{\lambda^{+}\right\},\left\{\lambda^{0}\right\}\right)$. If $\lambda_{j}$ are real, then $I_{k}\left(\left\{\lambda_{j}\right\}\right)$ is a real, bounded, symmetric function of all the $\lambda_{j}$. It is small in the limit of the weak coupling $c \rightarrow 0$, and in the limit of the strong coupling $c \rightarrow \infty: I_{k} \rightarrow c^{k-2}$ at $c \rightarrow 0 ; I_{k} \rightarrow c^{2-k}$ at $c \rightarrow \infty$. Note that $I_{k} \neq 0$ at $k \geqq 2$. The Fourier coefficients have similar properties

$$
\begin{array}{rlrl}
\mathscr{A}_{k}^{n} & \rightarrow c^{k-2} ; \mathscr{A}_{k}^{n} & \rightarrow c^{2-k} . \\
c & \rightarrow 0 & c & \rightarrow \infty
\end{array}
$$

The irreducible parts $I_{k}$ for small $k$ can be elementary evaluated either in the frame of coordinate Bethe's Ansatz, or in the frame of QISM. Below we shall need $k=2,3$ :

$$
I_{2}\left(\lambda_{1}, \lambda_{2}\right)=\frac{2}{\left(\lambda_{1}-\lambda_{2}\right)^{2}}\left(\frac{\lambda_{1}-\lambda_{2}+i c}{\lambda_{1}-\lambda_{2}-i c}\right)\left[1-e^{-i x\left(\lambda_{1}-\lambda_{2}\right)}\right]+\left(\lambda_{1}^{\prime} \leftrightarrow \lambda_{2}\right) .
$$

The Fourier coefficient $\mathscr{A}_{2}^{1}$ is equal to

$$
\mathscr{A}_{2}^{1}\left(\lambda_{1} \lambda_{2}\right)=-2\left(\lambda_{1}-\lambda_{2}+i c\right) /\left(\lambda_{1}-\lambda_{2}-i c\right)\left(\lambda_{1}-\lambda_{2}\right)^{2} \text {. }
$$

For $k=3$ we have

$$
I_{3}\left(\lambda_{1} \lambda_{2} \lambda_{3}\right)=\sum_{P}\left[e^{-i x\left(\lambda_{P_{1}}-\lambda_{P_{2}}\right)}-1\right] \mathscr{A}_{3}^{1}\left(\lambda_{P_{1}} \lambda_{P_{2}} \lambda_{P_{3}}\right)
$$

Here the sum is over permutations $P$ of $\lambda_{1} \lambda_{2} \lambda_{3}$. The Fourier coefficient $\mathscr{A}_{3}^{1}$ is equal to $\left(\lambda_{j k}=\lambda_{j}-\lambda_{k}\right)$ :

$$
\mathscr{A}_{3}^{1}\left(\lambda_{1} \lambda_{2} \lambda_{3}\right)=\frac{8 c}{\lambda_{12}^{2}}\left(\frac{\lambda_{12}+i c}{\lambda_{12}-i c}\right)\left[\frac{\lambda_{32}}{\lambda_{31}}+\frac{\lambda_{31}}{\lambda_{32}}\right] \frac{1}{\left(\lambda_{31}+i c\right)\left(\lambda_{23}+i c\right)} .
$$

To calculate the contribution of the irreducible part in the correlation function it must be "dressed." Let us define the dressing transformation. It is constructed by means of the function $P_{n}\left(t,\left\{\lambda^{+}\right\}_{n},\left\{\lambda^{-}\right\}_{n}\right)$, which depends on $(2 n+1)$ arguments. It is defined in a unique way by the dressing equation

$$
2 \pi P_{n}(t)=\left\{\prod_{j=1}^{n}\left(\frac{\lambda_{j}^{+}-t+i c}{\lambda_{j}^{+}-t-i c}\right)\left(\frac{\lambda_{j}^{-}-t-i c}{\lambda_{j}^{-}-t+i c}\right)\right\} \exp \left\{\int_{-q}^{q} K(t, s) P_{n}(s) d s\right\}-1
$$


and by inequality $\operatorname{Re} P_{n}\left(t,\left\{\lambda^{+}\right\},\left\{\lambda^{-}\right\}\right) \leqq 0$. The set $\left\{\lambda^{+}\right\}_{n}$ as well as the set $\left\{\lambda^{-}\right\}_{n}$ contains $n$ elements $\lambda_{j}^{+}$and $\lambda_{j}^{-}$correspondingly. Each of the arguments belongs to the interval $[-q, q],-q \leqq t \leqq q ;-q \leqq \lambda_{j}^{+} \leqq q ;-q \leqq \lambda_{j}^{-} \leqq q$. The properties of the $P_{n}$ which will be used below are as follows: 1) It is simply changed under the complex conjugation $P_{n}^{*}\left(t,\left\{\lambda^{+}\right\},\left\{\lambda^{-}\right\}\right)=P_{n}\left(t,\left\{\lambda^{-}\right\},\left\{\lambda^{+}\right\}\right)$. 2) The function $P_{n}$ is a symmetric function of all $\lambda_{j}^{+}$and of all $\lambda_{j}^{-}$separately. 3) The function $P_{n-1}$ is the special case of $P_{n}$; indeed at $\lambda_{n}^{+}=\lambda_{n}^{-} ; P_{n}=P_{n-1}$. If the whole set coincides $\lambda_{j}^{+}=\lambda_{j}^{-}, j=1, \ldots, n$, then $P_{n}=0$. 4) In the strong coupling limit $c \rightarrow \infty$ the function $P_{n}$ decreases

$$
P_{n}\left(t,\left\{\lambda^{+}\right\},\left\{\lambda^{-}\right\}\right)=\frac{i}{\pi c}\left(1+\frac{2 q}{\pi c}\right) \sum_{j=1}^{n}\left(\lambda_{j}^{-}-\lambda_{j}^{+}\right)-\frac{1}{\pi c^{2}}\left[\sum_{j=1}^{n}\left(\lambda_{j}^{+}-\lambda_{j}^{-}\right)\right]^{2} .
$$

5) At $c \rightarrow 0$ function $P_{n}$ goes to zero almost every where except the regions $\left|\lambda_{j}-t\right| \leqq c$, $P_{n}$ being bounded in these regions. 6) At any $c$ function $P_{n}$ is bounded in the domain of its definition: $\left.\left|P_{n}\left(t,\left\{\lambda^{+}\right\},\left\{\lambda^{-}\right\}\right)\right| \leqq 1 / \pi .7\right)$ For $\left\{\lambda^{+}\right\} \neq\left\{\lambda^{-}\right\}$and $0<c<\infty$, function $\operatorname{Re} P_{n} \neq 0$ in the domain of its definition.

Let us introduce also another function $p_{n}\left(\left\{\lambda^{+}\right\}_{n},\left\{\lambda^{-}\right\}_{n}\right)$. It depends on $2 n$ arguments. This function is defined in the following way

$$
p_{n}\left(\left\{\lambda^{+}\right\},\left\{\lambda^{-}\right\}\right)=i \sum_{j=1}^{n}\left(\lambda_{j}^{-}-\lambda_{j}^{+}\right)+\int_{-q}^{q} d t P_{n}\left(t,\left\{\lambda^{+}\right\},\left\{\lambda^{-}\right\}\right) .
$$

The dressing transformation $\mathbf{d}$ acts on the irreducible part (2.3) as follows: d: $I_{k}(\{\lambda\}) \rightarrow I_{k}^{d}(\{\lambda\})$. Here the dressed irreducible part is equal to:

$$
I_{k}^{d}\left(\left\{\lambda_{j}\right\}\right)=\sum_{\{\lambda\}=\left\{\lambda^{+}\right\} \cup\left\{\lambda^{-}\right\} \cup\left\{\lambda^{0}\right\}} e^{x p_{n}\left(\left\{\lambda^{+}\right\},\left\{\lambda^{-}\right\}\right)} \mathscr{A}_{k}^{n}\left(\left\{\lambda^{+}\right\},\left\{\lambda^{-}\right\},\left\{\lambda^{0}\right\}\right)
$$

Here the sum is taken over the same partitions as in (2.3).

So the dressing transformation acts only on the exponents by means of replacement $\exp \left\{-i x \sum\left(\lambda^{+}-\lambda^{-}\right)\right\} \mapsto \exp \left\{x p_{n}\left(\left\{\lambda^{+}\right\},\left\{\lambda^{-}\right\}\right)\right\}$, the Fourier coefficients $\mathscr{A}$ remaining invariable. In this way we have defined the dressing transformation. It is important that the function $I_{k}^{d}(\{\lambda\})$ is real, symmetric and a bounded function of all the $\lambda_{j}$.

Now everything is ready to describe the decomposition of the correlation functions. The contribution of the $k$-particle processes $\Gamma_{k}$ can be obtained by the integration of the $I_{k}^{d}(\{\lambda\})$ over all the variables $\lambda_{j}$ with the weight $\omega(\lambda)$ :

$$
\Gamma_{k}=\frac{1}{k !} \int_{-q}^{q} \prod_{j=1}^{k}\left[\frac{\omega\left(\lambda_{j}\right) d \lambda_{j}}{2 \pi}\right] I_{k}^{d}\left(\left\{\lambda_{j}\right\}\right) .
$$

Here the weight $\omega(\lambda)$ is equal to

$$
\omega(\lambda)=\exp \left\{\frac{-1}{2 \pi} \int_{-q}^{q} K(\lambda, \mu) d \mu\right\} .
$$

Its variation is bounded $1 / e \leqq \omega(\lambda)<\omega_{0}$,

$$
0<\omega_{0}=\exp \left\{\frac{-2 c q}{\pi\left(c^{2}+4 q^{2}\right)}\right\}<1 .
$$


The correlation function of the currents is equal to

$$
\frac{\langle\Omega|j(x) j(0)| \Omega\rangle}{\langle\Omega \mid \Omega\rangle}=\left[\int_{-q}^{q} \rho(\lambda) d \lambda\right]^{2}+\frac{1}{2} \frac{\partial^{2}}{\partial x^{2}} \sum_{k=2}^{\infty} \Gamma_{k} .
$$

Here $x>0$. Note that the first term gives the long wave asymptotics (1.6). The formulae (2.4), (2.9), (2.11), (2.12), (2.13) show that $\Gamma_{k} \rightarrow c^{2-k}$ at $c \rightarrow \infty$. So decomposition (2.15) is similar to the $1 / c$ expansion, which can be extracted from our decomposition by means of elementary calculation. Nevertheless the $1 / c$ expansion gives nonuniform approximation in the distance due to the polynomial contributions. The decomposition (2.15) avoids this defect, it gives the uniform approximation in $x$. Formula (2.15) is the main result of the paper. The whole paper is devoted to its derivation.

Let us calculate first two terms in decomposition (2.15).

To calculate $\Gamma_{2}$ one needs $I_{2}(2.5)$. The $I_{2}^{d}$ is equal to

$$
I_{2}^{d}\left(\lambda_{1} \lambda_{2}\right)=\frac{2}{\lambda_{12}^{2}}\left(\frac{\lambda_{12}+i c}{\lambda_{12}-i c}\right)\left[1-e^{x p_{2}\left(\lambda_{1} \lambda_{2}\right)}\right]+\left(\lambda_{1} \leftrightarrow \lambda_{2}\right) .
$$

The two particle contribution $\Gamma_{2}$ is equal to

$$
\Gamma_{2}=\frac{1}{2 \pi^{2}} \int_{-q}^{q} \omega\left(\lambda_{1}\right) d \lambda_{1} \int_{-q}^{q} \omega\left(\lambda_{2}\right) d \lambda_{2}\left(\frac{\lambda_{1}-\lambda_{2}+i c}{\lambda_{1}-\lambda_{2}-i c}\right)\left[\frac{1-\exp \left\{x p_{2}\left(\lambda_{1}, \lambda_{2}\right)\right\}}{\left(\lambda_{1}-\lambda_{2}\right)^{2}}\right] .
$$

The symmetry of (2.16) shows that the principal value of the integral must be taken. Contribution $\Gamma_{3}$ is generated by $I_{3}(2.6),(2.7)$

$$
\begin{aligned}
\Gamma_{3}= & \frac{c}{\pi^{3}} \int_{-q}^{q} \omega\left(\lambda_{1}\right) d \lambda_{1} \int_{-q}^{q} \omega\left(\lambda_{2}\right) d \lambda_{2} \int_{-q}^{q} \omega\left(\lambda_{3}\right) d \lambda_{3}\left(\frac{\lambda_{1}-\lambda_{2}+i c}{\lambda_{1}-\lambda_{2}-i c}\right) \\
& \times\left(\frac{\lambda_{3}-\lambda_{2}}{\lambda_{3}-\lambda_{1}}+\frac{\lambda_{3}-\lambda_{1}}{\lambda_{3}-\lambda_{2}}\right) \frac{\left[\exp \left\{x p_{2}\left(\lambda_{1} \lambda_{2}\right)\right\}-1\right]}{\left(\lambda_{1}-\lambda_{2}\right)^{2}\left(\lambda_{3}-\lambda_{1}+i c\right)\left(\lambda_{2}-\lambda_{3}+i c\right)} .
\end{aligned}
$$

The principal value of the integral must be taken. Let us write down the correlation function (2.15):

$$
\begin{aligned}
& \frac{\langle\Omega|j(x) j(0)| \Omega\rangle}{\langle\Omega \mid \Omega\rangle}=\left[\int_{-q}^{q} \rho(\lambda) d \lambda\right]^{2}-\int_{-q}^{q} \frac{d^{2} \lambda}{(2 \pi)^{2}} \omega\left(\lambda_{1}\right) \omega\left(\lambda_{2}\right)\left(\frac{\lambda_{1}-\lambda_{2}+i c}{\lambda_{1}-\lambda_{2}-i c}\right) \\
& \times\left[\frac{p_{2}\left(\lambda_{1} \lambda_{2}\right)}{\lambda_{1}-\lambda_{2}}\right]^{2} e^{x p_{2}\left(\lambda_{1} \lambda_{2}\right)}+\frac{c}{2 \pi^{3}} \int_{-q}^{q} d^{3} \lambda \omega\left(\lambda_{1}\right) \omega\left(\lambda_{2}\right) \omega\left(\lambda_{3}\right)\left[\frac{p_{2}\left(\lambda_{1} \lambda_{2}\right)}{\lambda_{1}-\lambda_{2}}\right]^{2} \\
& \quad \times\left(\frac{\lambda_{12}+i c}{\lambda_{12}-i c}\right)\left(\frac{\lambda_{32}}{\lambda_{31}}+\frac{\lambda_{31}}{\lambda_{32}}\right) \frac{\exp \left\{x p_{2}\left(\lambda_{1} \lambda_{2}\right)\right\}}{\left(\lambda_{31}+i c\right)\left(\lambda_{23}+i c\right)}+O\left(\frac{1}{c^{2}}\right) .
\end{aligned}
$$

Note that $p_{2}\left(\lambda_{1} \lambda_{2}\right)$ tends to zero as $\lambda_{1} \rightarrow \lambda_{2}: p_{2}\left(\lambda_{1} \lambda_{2}\right) \rightarrow\left(\lambda_{1}-\lambda_{2}\right) p\left(\lambda_{1}+\lambda_{2}\right)$. In the strong coupling limit $c \rightarrow \infty$ the correction to this expression is of order $1 / c^{2}$ at any 
value of $x>0$. In this limit the expression is simplified:

$$
\begin{aligned}
\frac{\langle\Omega|j(x) j(0)| \Omega\rangle}{\langle\Omega \mid \Omega\rangle}= & \left(\frac{q}{\pi}\right)^{2}+\frac{4}{c}\left(\frac{q}{\pi}\right)^{3}-\left(1+\frac{2}{c} \frac{\partial}{\partial x_{r}}\right)\left(\frac{\sin q x_{r}}{\pi x_{r}}\right)^{2} \\
& +\frac{2}{c \pi^{2}} \frac{\partial}{\partial x}\left[\frac{\sin q x}{\pi x} \int_{-q}^{q} d \lambda \sin \lambda x \ln \left(\frac{q+\lambda}{q-\lambda}\right)\right] \\
& -\frac{8 q}{\pi c}\left(\frac{\sin q x}{\pi x}\right)^{2}+O\left(1 / c^{2}\right) .
\end{aligned}
$$

Here $x_{r}=(1+2 q / \pi c) x$. One can easily calculate the two initial terms in the $1 / c$ expansion and verify that the $1 / c$ expansion gives a nonuniform approximation in the distance. In the same way one can calculate any term in the expansion (2.15). The proof of (2.15) goes as follows. In the next sect. we derive the formula, expressing the mean value of the operator $\mathbf{Q}_{1}^{2}(2.2)$ in terms of irreducible parts. We shall use the two-site generalized model of the paper [1]. In Sects. 4, 5 we shall pass to the thermodynamic limit in this formula and complete the proof of (2.15).

\section{The Expression of the Mean Value $\left\langle\mathbf{Q}_{1}^{2}\right\rangle$ in terms of Irreducible Parts}

In this sect. we derive the formula expressing the mean value $\left\langle Q_{1}^{2}\right\rangle_{N}$ in terms of irreducible parts $I_{k}, 2 \leqq k \leqq N$. We use the two-site generalized model, see Sect. 2 of the paper [1]. Let us consider, first of all, the mean value of the identity operator (8.1) [1] with respect to the eigenfunction:

$$
\langle\mathbf{1}\rangle_{N}=\frac{\left\langle 0\left|\prod_{j=1}^{N} C\left(\lambda_{j}\right) \prod_{j=1}^{N} B\left(\lambda_{j}\right)\right| 0\right\rangle}{c^{N}\left\{\prod_{j=1}^{N} a\left(\lambda_{j}\right) d\left(\lambda_{j}\right)\right\} \prod_{j \neq k} f_{j k}}=\operatorname{det}_{N}\left(\varphi^{\prime}\right) .
$$

Here we denote $f_{j k}=f\left(\lambda_{j} \lambda_{k}\right)=1+i c /\left(\lambda_{j}-\lambda_{k}\right)$ and

$$
\varphi_{j}=i \ln r\left(\lambda_{j}\right)+i \sum_{\substack{k=1 \\ k \neq j}}^{N} \ln \left(f_{j k} / f_{k j}\right) ; r(\lambda)=\frac{a(\lambda)}{d(\lambda)} .
$$

The explicit expression for the $N \times N$-matrix $\varphi_{j k}^{\prime}=\partial \varphi_{j} / \partial \lambda_{k}$ is presented in Appendix A. So $N$ in the expression $\operatorname{det}_{N} \varphi^{\prime}$ denotes the dimension of matrix $\varphi^{\prime}$. For $N=0$ we put $\operatorname{det}_{0} \varphi^{\prime}=1$. The momenta $\lambda_{j}$ satisfy the equations

$$
r\left(\lambda_{j}\right) \prod_{\substack{k=1 \\ k \neq j}}^{N}\left(f_{j k} / f_{k j}\right)=1 .
$$

The values $z_{j}=i \partial\left\{\ln r\left(\lambda_{j}\right) / \partial \lambda_{j}\right\}$ can be regarded as independent variables $[1,9,18]$.

Note. For the NS model $r(\lambda)=\exp \{-i \lambda L\}, z_{j}=L$. For $N=0$ we put $\operatorname{det}_{0} \varphi^{\prime}=1$. The most important properties of the determinant are as follows (see [9]): 
1) It is a symmetric function invariant under replacement of pairs $\left(\lambda_{k}, z_{k}\right) \leftrightarrow\left(\lambda_{j}, z_{j}\right)$. 2) It is a linear function in $z_{N}$. 3) The coefficient at $z_{N}$ is equal to

$$
\frac{\partial}{\partial z_{N}} \operatorname{det}_{N}\left(\varphi^{\prime}\right)=\operatorname{det}_{(N-1)}\left(\tilde{\varphi}^{\prime}\right) .
$$

The matrix $\left(\tilde{\varphi}^{\prime}\right)_{j k}=\partial \tilde{\varphi}_{j} / \partial \lambda_{k} ; j, k=1, \ldots,(N-1)$ is defined by means of $\tilde{\varphi}_{j}$, which is given by (3.2) with the replacement $N \rightarrow(N-1)$ and $r(\lambda) \rightarrow \tilde{r}(\lambda)=r(\lambda) f\left(\lambda \lambda_{N}\right) / f\left(\lambda_{N} \lambda\right)$. It means that $z_{j}$ is replaced by $\tilde{z}_{j}$

$$
\tilde{z}_{j}=z_{j}+K_{j N}
$$

Here we use the notation $K_{j \ell}=K\left(\lambda_{j} \lambda_{\ell}\right)$, see(1.3). The Jacobian at the right-hand side of (3.4) does not contain $z_{N} ; \lambda_{N}$ is included in $\tilde{r}(\lambda)$. 4) The determinant is equal to zero at all $z_{j}=0$ and $\lambda_{j}$ fixed (for $N \geqq 1$ )

$$
\operatorname{det}_{N} \varphi^{\prime}=\delta_{0}^{N}, \quad \text { at } \quad z_{j}=0 .
$$

5) For $N=1$

$$
\operatorname{det}_{1} \varphi^{\prime}=z_{1} .
$$

It was noted in paper [9] that the left-hand side of (3.1) has the same properties. It permits us to prove (3.1) by induction in $N$. One can say that the irreducible part of the identity operator $I_{N}^{(0)}=\delta_{0}^{N}$, see (7.6) [1], generates formula (3.1).

In the two-site generalized model, the variables $z_{j}$ are equal to:

$$
z_{j}=x_{j}+y_{j}
$$

Here $\quad x_{j}=i \partial\left[\ln \ell\left(\lambda_{j}\right)\right] / \partial \lambda_{j} ; \quad y_{j}=i \partial\left[\ln m\left(\lambda_{j}\right)\right] / \partial \lambda_{j} \quad$ and $\quad \ell(\lambda)=a_{1}(\lambda) / d_{1}(\lambda)$; $m(\lambda)=a_{2}(\lambda) / d_{2}(\lambda), r(\lambda)=\ell(\lambda) m(\lambda)$.

Note. For the NS model $\ell(\lambda)=\exp \{-i \lambda x\}, x_{j}=x, y_{j}=L-x$.

Our aim is to express the $\operatorname{det} \varphi^{\prime}$ in terms of $x$ and $y$. The following definitions are useful. Let use consider a partition of the set $\left\{\hat{\lambda}_{j}\right\}_{N}$ into two disjoint subsets $\left\{\lambda^{x}\right\}$ and $\left\{\lambda^{y}\right\}$

$$
\left\{\lambda_{j}\right\}_{N}=\left\{\lambda^{x}\right\} \cup\left\{\lambda^{y}\right\},\left\{\lambda^{x}\right\} \cap\left\{\lambda^{y}\right\}=\varnothing .
$$

Here card $\left\{\lambda^{x}\right\}=n_{x}, \operatorname{card}\left\{\lambda^{y}\right\}=n_{y}, \operatorname{card}\left\{\lambda_{j}\right\}=N, n_{x}+n_{y}=N$. We write the number of elements as the subindex of the set, for example, $\left\{\lambda^{x}\right\}_{n_{x}}$. The values $\varphi^{x}$ and $\varphi^{y}$ are defined by:

$$
\begin{aligned}
& \varphi_{j}^{x}=i \ln \ell\left(\lambda_{j}^{x}\right)+\sum_{\substack{\lambda_{k}^{x} \in\left\{\lambda^{x}\right\} \\
k \neq j}} i \ln \frac{f\left(\lambda_{j}^{x} \lambda_{k}^{x}\right)}{f\left(\lambda_{k}^{x} \lambda_{j}^{x}\right)}, \\
& \varphi_{j}^{y}=i \ln m\left(\lambda_{j}^{y}\right)+\sum_{\lambda_{k}^{y} \in\left\{\lambda_{y}\right\}, k \neq j} i \ln \frac{f\left(\lambda_{j}^{y}, \lambda_{k}^{y}\right)}{f\left(\lambda_{k}^{y}, \lambda_{j}^{y}\right)} .
\end{aligned}
$$

The number of $\varphi_{j}^{x}$ is equal to $n_{x}$, the number of $\varphi_{j}^{y}$ is equal to $n_{y}$. Now we can 
introduce two Jacobians

$$
\begin{aligned}
& \operatorname{det}_{n_{x}}\left(\varphi_{x}^{\prime}\right),\left(\varphi_{x}^{\prime}\right)_{j k}=\partial \varphi_{j}^{x} / \partial \lambda_{k}^{x}, \\
& \operatorname{det}_{n_{y}}\left(\varphi_{y}^{\prime}\right),\left(\varphi_{y}^{\prime}\right)_{j k}=\partial \varphi_{j}^{y} / \partial \lambda_{k}^{y} .
\end{aligned}
$$

It should be noted that these Jacobians are exactly of the same kind as $\operatorname{det} \varphi^{\prime}$. They have the same five properties: 1) Determinant (3.12) is invariant under the replacement of pairs $\left(\lambda_{k} ; x_{k}\right) \leftrightarrow\left(\lambda_{j} ; x_{j}\right)$, and (3.13) is invariant under the replacement of $\left(\lambda_{k} ; y_{k}\right) \leftrightarrow\left(\lambda_{j} ; y_{j}\right)$.2) Determinant (3.12) is a linear function in $x_{n_{x}} ;(3.13)$ is a linear function in $y_{n_{y}}$. 3) The coefficients are equal to

$$
\frac{\partial}{\partial x_{n_{x}}} \operatorname{det}_{n_{x}}\left(\varphi_{x}^{\prime}\right)=\operatorname{det}_{\left(n_{x}-1\right)}\left(\tilde{\varphi}_{x}^{\prime}\right)
$$

and similarly $\partial \operatorname{det}_{n}\left(\varphi_{y}^{\prime}\right) / \partial y_{n}=\operatorname{det}_{(n-1)}\left(\tilde{\varphi}_{y}^{\prime}\right)$, with

$$
\tilde{x}_{j}=x_{j}+K\left(\lambda_{J}^{x} \lambda_{n_{x}}^{x}\right) ; \quad \tilde{y}_{j}=y_{J}+K\left(\lambda_{j}^{y} \lambda_{n_{y}}^{y}\right) .
$$

4) At $x_{j}=0$ Jacobian (3.12) is equal to zero for $n \geqq 1$ :

$$
\left.\operatorname{det}_{n}\left(\varphi_{x}^{\prime}\right)\right|_{x_{j}=0}=\delta_{0}^{n},\left.\quad \operatorname{det}_{n}\left(\varphi_{y}^{\prime}\right)\right|_{y_{j}=0}=\delta_{0}^{n}, \quad j=1, \ldots, n .
$$

5) In the scalar case they are equal to

$$
\operatorname{det}_{1}\left(\varphi_{x}^{\prime}\right)=x_{1}, \operatorname{det}_{1}\left(\varphi_{y}^{\prime}\right)=y_{1} .
$$

Now everything is ready to prove the following

Theorem 1. The determinant (3.1) is expressed in terms of the determinants (3.12), (3.13) by the following formula

$$
\operatorname{det}_{N}\left(\varphi^{\prime}\right)=\sum_{\left\{\lambda_{j}\right\}=\left\{\lambda_{x}\right\} \cup\left\{\lambda_{y}\right\}} \operatorname{det}_{n_{x}}\left(\varphi_{x}^{\prime}\right) \operatorname{det}_{n_{y}}\left(\varphi_{y}^{\prime}\right) .
$$

The summation is performed over all the partitions (3.9).

The proof goes by induction in $N$, starting from $N=1$ (compare (3.7), (3.8) and (3.17)). Assume that (3.18) is already proved for $N=1, \ldots, M-1$. We shall show that then it is valid for $N=M$. Let us consider the value

$$
\begin{aligned}
& \Delta_{M}=\operatorname{det}_{M}\left(\varphi^{\prime}\right)-\sum_{\left\{\lambda_{j}\right\}_{M}=\left\{\lambda^{x}\right\} \cup\left\{\lambda^{y}\right\}} \operatorname{det}_{n_{x}}\left(\varphi_{x}^{\prime}\right) \operatorname{det}_{n_{y}}\left(\varphi_{y}^{\prime}\right), \\
& n_{x}+n_{y}=M
\end{aligned}
$$

It is a linear function in $x_{M}$. The derivative with respect to $x_{M}$ is equal to

$$
\begin{aligned}
& \frac{\partial \Delta_{M}}{\partial x_{M}}=\operatorname{det}_{(M-1)}\left(\hat{\varphi}^{\prime}\right)-\sum_{\left\{\lambda_{j}\right\}_{M-1}=\left\{\lambda_{x}\right\} \cup\left\{\lambda_{y}\right\}} \operatorname{det}_{n_{x}}\left(\tilde{\varphi}_{x}^{\prime}\right) \operatorname{det}_{n_{y}}\left(\varphi_{y}^{\prime}\right), \\
& n_{x}+n_{y}=M-1
\end{aligned}
$$

see (3.4) and (3.14). The right-hand side is equal to zero due to inductive assumption and (3.5), (3.15), (3.8). So $\partial \Delta_{M} / \partial x_{M}=0$. Due to the symmetry $\partial \Delta_{M} / \partial x_{j}=0$, $j=1, \ldots, M$. Similarly one can prove that $\partial \Delta_{M} / \partial y_{j}=0$. So $\Delta_{M}$ is $x_{j}$ and $y_{j}$ 
independent. But $\Delta_{M}=0$ at $x_{j}=y_{j}=0$, due to (3.6),(3.16). So $\Delta_{M}=0$ identically, and (3.18) is valid for $N=M$. This completes the proof of the theorem. It should be noted that the s.t.e. (3.3) was not used in the proof. All the proofs in this sect. will be of this kind.

Let us consider now the operator of number of particles in the first site of the lattice $\mathbf{Q}_{1}(2.18)$ [1]. The mean value of the operator $\mathbf{Q}_{1}$ :

$$
\left\langle\mathbf{Q}_{1}\right\rangle_{N}=\frac{\left\langle 0\left|\prod_{j=1}^{N} C\left(\lambda_{j}\right) \mathbf{Q}_{1} \prod_{j=1}^{N} B\left(\lambda_{j}\right)\right| 0\right\rangle}{c^{N}\left\{\prod_{j=1}^{N} a\left(\lambda_{j}\right) d\left(\lambda_{j}\right)\right\} \prod_{j \neq k} f_{j k}}
$$

with respect to the $N$-particle eigenvector (3.3) depends on $4 N$ variables $\left\langle\mathbf{Q}_{1}\right\rangle_{N}=\left\langle\mathbf{Q}_{1}\right\rangle_{N}\left(\left\{\lambda_{j}\right\},\left\{x_{j}\right\},\left\{y_{j}\right\},\left\{\ell_{j}\right\}\right)$, see (8.2) [1]. We remind the reader of the properties of $\left\langle\mathbf{Q}_{1}\right\rangle$, see Sect. 8 of paper [1]. 1) It is invariant under replacement of $\left.\left(\lambda_{k}, x_{k}, y_{k}, \ell_{k}\right) \leftrightarrow\left(\lambda_{j}, x_{j}, y_{j}, \ell_{j}\right) .2\right)$ It is a linear function on $x_{N}$ and $\left.y_{N} .3\right)$ The coefficient at $y_{N}$ is equal to

$$
\frac{\partial}{\partial y_{N}}\left\langle\mathbf{Q}_{1}\right\rangle_{N}=\left\langle\mathbf{Q}_{1}\right\rangle_{(N-1}\left(\left\{\lambda_{j}\right\}_{(N-1)},\left\{x_{j}\right\}_{(N-1)},\left\{y_{j}+K_{j N}\right\}_{(N-1)},\left\{\ell_{j}\right\}_{(N-1)}\right) .
$$

The coefficient at $x_{N}$ is equal to

$$
\frac{\partial}{\partial x_{N}}\left\langle\mathbf{Q}_{1}\right\rangle_{N}=\left\langle\mathbf{Q}_{1}+\mathbf{1}\right\rangle_{(N-1)}\left(\left\{\lambda_{j}\right\},\left\{x_{j}+K_{j N}\right\},\left\{y_{j}\right\},\left\{\ell_{j} \frac{f_{j N}}{f_{N j}}\right\}\right) \text {. }
$$

Here we denote $\left\langle\mathbf{Q}_{1}+\mathbf{1}\right\rangle=\left\langle\mathbf{Q}_{1}\right\rangle+\langle\mathbf{1}\rangle$. At the right-hand side of (3.20), (3.21) $j=1, \ldots,(N-1)$, so the variables $x_{N}, y_{N}$ and $\ell_{N}$ are absent. 4) The meanvalue is equal to zero at $x_{j}=y_{j}=0$ and $\lambda, \ell$ fixed: $\left\langle\mathbf{Q}_{1}\right\rangle\left(\left\{\lambda_{j}\right\},\{0\},\{0\},\left\{\ell_{j}\right\}\right)=0$. 5) In the one particle sector $\left\langle\mathbf{Q}_{1}\right\rangle_{1}=x_{1}$. By means of these properties one proves the following:

Theorem 2. The mean value of operator $\mathbf{Q}_{1}$ is expressed in terms of the determinants (3.12), (3.13) by the following formula:

$$
\left\langle\mathbf{Q}_{1}\right\rangle_{N}=\sum_{\left\{\lambda_{j}\right\}=\left\{\lambda \lambda^{x}\right\} \cup\{\lambda y\}} n_{x} \operatorname{det}_{n_{x}}\left(\varphi_{x}^{\prime}\right) \operatorname{det}_{n_{y}}\left(\varphi_{y}^{\prime}\right) .
$$

Here we use the same notations as in Theorem 1. The proof is similar to that of Theorem 1.

The normalized meanvalue of $\mathbf{Q}_{1}$ is equal to

$$
\begin{gathered}
\frac{\left\langle 0\left|\prod_{j=1}^{N} C\left(\lambda_{j}\right) \mathbf{Q}_{1} \prod_{j=1}^{N} B\left(\lambda_{j}\right)\right| 0\right\rangle}{\left\langle 0\left|\prod_{j=1}^{N} C\left(\lambda_{j}\right) \prod_{j=1}^{N} B\left(\lambda_{j}\right)\right| 0\right\rangle} \equiv \frac{\left\langle\mathbf{Q}_{1}\right\rangle_{N}}{\langle\mathbf{1}\rangle_{N}} \\
=\sum_{\left\{\lambda_{j}\right\}=\left\{\lambda^{x}\right\} \cup\left\{\lambda^{\nu}\right\}} n_{x} \frac{\operatorname{det}_{n_{x}}\left(\varphi_{x}^{\prime}\right) \operatorname{det}_{n_{y}}\left(\varphi_{y}^{\prime}\right)}{\operatorname{det}_{N}\left(\varphi^{\prime}\right)} .
\end{gathered}
$$


One can say that formula (3.22) is generated by the irreducible part of the identity operator.

At last let us consider operator $\mathbf{Q}_{1}^{2}$. The correlation function can be expressed in terms of it (2.1). Let us denote the mean value of the operator $\mathbf{Q}_{1}^{2}$ with respect to the $N$-particle eigenfunction by

$$
\frac{\left\langle 0\left|\prod_{j=1}^{N} C\left(\lambda_{j}\right) \mathbf{Q}_{1}^{2} \prod_{j=1}^{N} B\left(\lambda_{j}\right)\right| 0\right\rangle}{c^{N} \prod_{j=1}^{N} a\left(\lambda_{j}\right) d\left(\lambda_{j}\right) \prod_{j \neq k}^{N} f_{j k}} \equiv\left\langle\mathbf{Q}_{1}^{2}\right\rangle_{N}\left(\left\{\lambda_{j}\right\},\left\{x_{j}\right\},\left\{y_{j}\right\},\left\{\ell_{j}\right\}\right) .
$$

see(8.7) [1]. We remind the reader of the properties of $\left\langle\mathbf{Q}_{1}^{2}\right\rangle$, see Sect. 8 of [1]. 1) It is invariant under replacement of $\left(\lambda_{k}, x_{k}, y_{k}, \ell_{k}\right) \leftrightarrow\left(\lambda_{j} x_{j} y_{j} \ell_{j}\right)$. 2) It is a linear function on $x_{N}$ and $\left.y_{N} .3\right)$ The coefficients are equal to

$$
\begin{aligned}
& \frac{\partial}{\partial y_{N}}\left\langle\mathbf{Q}_{1}^{2}\right\rangle_{N}\left(\left\{\lambda_{j}\right\},\left\{x_{j}\right\},\left\{y_{j}\right\},\left\{\ell_{j}\right\}\right) \\
& \quad=\left\langle\mathbf{Q}_{1}^{2}\right\rangle_{(N-1)}\left(\left\{\lambda_{j}\right\},\left\{x_{j}\right\},\left\{y_{j}+K_{j N}\right\},\left\{\ell_{j}\right\}\right), \\
& \frac{\partial}{\partial x_{N}}\left\langle\mathbf{Q}_{1}^{2}\right\rangle_{N}\left(\left\{\lambda_{j}\right\},\left\{x_{j}\right\},\left\{y_{j}\right\},\left\{\ell_{j}\right\}\right) \\
& \quad=\left\langle\left(\mathbf{Q}_{1}+1\right)^{2}\right\rangle_{(N-1)}\left(\left\{\lambda_{j}\right\},\left\{x_{j}+K_{j N}\right\},\left\{y_{j}\right\},\left\{\ell_{j} \frac{f_{j N}}{f_{N j}}\right\}\right) .
\end{aligned}
$$

Here $\left\langle\left(\mathbf{Q}_{1}+\mathbf{1}\right)^{2}\right\rangle=\left\langle\mathbf{Q}_{1}^{2}\right\rangle+2\left\langle\mathbf{Q}_{1}\right\rangle+\langle\mathbf{1}\rangle$. In the right-hand side of (3.25), (3.26) $j=1, \ldots, N-1$, so $x_{N}, y_{N}$ and $\ell_{N}$ are absent. 4) The mean value $\left\langle\mathbf{Q}_{1}^{2}\right\rangle$ at $x_{j}=y_{j}=$ $0(j=1, \ldots, N)$ is equal to the irreducible part (7.11) [1].

$$
\begin{aligned}
& \left\langle\mathbf{Q}_{1}^{2}\right\rangle_{N}\left(\left\{\lambda_{j}\right\},\{0\},\{0\},\left\{\ell_{j}\right\}\right)=I_{N}\left(\left\{\lambda_{j}\right\},\left\{\ell_{j}\right\}\right) \\
& =\sum_{\left\{\lambda_{j}\right\}=\left\{\lambda^{+}\right\} \cup\left\{\lambda^{-}\right\} \cup\left\{\lambda^{0}\right\}} \mathscr{A}_{N}^{n}\left(\left\{\lambda^{+}\right\},\left\{\lambda^{-}\right\},\left\{\lambda^{0}\right\}\right) \prod_{j=1}^{n} \ell\left(\lambda_{j}^{+}\right) \ell^{-1}\left(\lambda_{j}^{-}\right) .
\end{aligned}
$$

It is not equal to zero for $N \geqq 2$. 5) In the one-particle sector $\left\langle\mathbf{Q}_{1}^{2}\right\rangle_{1}=x_{1}$.

In this case the irreducible part of the identity operator and all the irreducible parts of the operator $\mathbf{Q}_{1}^{2}$ will give the contribution to the value $\left\langle\mathbf{Q}_{1}^{2}\right\rangle$. Let us introduce the value

$$
\left\langle\mathbf{Q}_{1}^{2}\right\rangle_{N}^{0}=\sum_{\{\lambda\}=\left\{\lambda^{x}\right\} \cup\left\{\lambda^{y}\right\}} n_{x}^{2} \operatorname{det}_{n_{x}}\left(\varphi_{x}^{\prime}\right) \operatorname{det}_{n_{y}}\left(\varphi_{y}^{\prime}\right),
$$

which gives the contribution of the irreducible part of the identity operator in $\left\langle\mathbf{Q}_{1}^{2}\right\rangle$. Each irreducible part $I_{k}$ generates the contribution to the $\left\langle\mathbf{Q}_{1}^{2}\right\rangle$. We shall denote it by $\mathscr{D}_{N}^{k}$

$$
\mathscr{D}_{N}^{k}\left(\{\lambda\}_{N}\right)=\sum_{\{\lambda\}_{N}=\left\{\lambda^{I}\right\}_{k} \cup\left\{\lambda^{\nu}\right\}_{N-k}} \square_{k, N}^{d}\left(\left\{\lambda^{I}\right\}_{k},\left\{\lambda^{v}\right\}_{N-k}\right) .
$$

The sum is taken over all the partitions of the set $\left\{\lambda_{j}\right\}$ into two disjoint subsets 
$\left\{\lambda^{I}\right\}$, and $\left\{\lambda^{v}\right\}$, card $\left\{\lambda^{I}\right\}=k$, card $\left\{\lambda^{v}\right\}=N-k$. The value $\mathbb{q}_{k, N}^{d}$ is equal to

$$
\begin{aligned}
& \mathbb{U}_{k, N}^{d}=\sum_{\left\{\lambda^{I}\right\}_{k}=\left\{\lambda^{+}\right\}_{n} \cup\left\{\lambda^{-}\right\}_{n} \cup\left\{\lambda^{0}\right\}_{k-2 n}}^{n \leqq[k / 2]} \mathscr{A}_{k}^{n}\left(\left\{\lambda^{+}\right\}_{n},\left\{\lambda^{-}\right\}_{n},\left\{\lambda^{0}\right\}_{k-2 n}\right) \\
& \times \mathbb{E}_{n, N-k}\left(\left\{\lambda^{+}\right\},\left\{\lambda^{-}\right\},\left\{\lambda^{\nu}\right\}_{N-k}\right) \prod_{j=1}^{n} \ell\left(\lambda_{j}^{+}\right) \ell^{-1}\left(\lambda_{j}^{-}\right) .
\end{aligned}
$$

Here the values $\mathscr{A}_{k}^{n}$ are the Fourier coefficients of the irreducible part (3.27). The sum is taken over the partitions of $\left\{\lambda^{I}\right\}$ into three disjoint subsets $\left\{\lambda^{+}\right\},\left\{\lambda^{-}\right\}$and $\left\{\lambda^{0}\right\}$; card $\left\{\lambda^{+}\right\}=\operatorname{card}\left\{\lambda^{-}\right\}=n$, card $\left\{\lambda^{0}\right\}=k-2 n$, like in the expression for the irreducible part (3.27). The value $\mathbb{E}_{n, N-k}$ is equal to

$$
\begin{aligned}
\mathbb{E}_{n, N-k}\left(\left\{\lambda^{+}\right\}_{n},\left\{\lambda^{-}\right\}_{n},\left\{\lambda^{\nu}\right\}_{N-k}\right) & \\
= & \sum_{\left\{\lambda^{v}\right\}_{N-k}=\left\{\lambda^{x}\right\} \cup\left\{\lambda^{y}\right\}} \operatorname{det}_{n_{x}}\left(\varphi_{x}^{\prime}\right) \operatorname{det}_{n_{y}}\left(\varphi_{y}^{\prime}\right) \prod_{i=1}^{n_{x}} \prod_{j=1}^{n}\left(\frac{f\left(\lambda_{j}^{+} \lambda_{i}^{x}\right)}{f\left(\lambda_{i}^{x} \lambda_{j}^{+}\right)} \frac{f\left(\lambda_{i}^{x} \lambda_{j}^{-}\right)}{f\left(\lambda_{j}^{-} \lambda_{i}^{x}\right)}\right), \\
& \mathbb{E}_{n, 0} \equiv 1 .
\end{aligned}
$$

The sum here is taken over the partitions of the set $\left\{\lambda^{v}\right\}$ into two disjoint subsets $\left\{\lambda^{x}\right\}$ and $\left\{\lambda^{y}\right\}$, card $\left\{\lambda^{x}\right\}=n_{x}, \operatorname{card}\left\{\lambda^{y}\right\}=n_{y}, n_{x}+n_{y}=N-k$ like in (3.9), (3.18), (3.22) and (3.28). So we define the expression $\mathscr{D}_{N}^{k}$. It is equal to the sum over the partition of $\left\{\lambda_{j}\right\}_{N}$ into five disjoint subsets $\{\lambda\}_{N}=\left\{\lambda^{I}\right\} \cup\left\{\lambda^{v}\right\},\left\{\lambda^{I}\right\}=\left\{\lambda^{+}\right\} \cup\left\{\lambda^{-}\right\} \cup\left\{\lambda^{0}\right\}$, $\left\{\lambda^{v}\right\}=\left\{\lambda^{x}\right\} \cup\left\{\lambda^{y}\right\}$. Now one can prove the following:

Theorem 3. The mean value of the operator $\mathbf{Q}_{1}^{2}$ is expressed in terms of the irreducible parts by the following formula

$$
\left\langle\mathbf{Q}_{1}^{2}\right\rangle_{N}=\left\langle\mathbf{Q}_{1}^{2}\right\rangle_{N}^{0}+\sum_{k=2}^{N} \mathscr{D}_{N}^{k} .
$$

The proof is similar to that of Theorem 1 .

Now let us normalize the mean value $\left\langle\mathbf{Q}_{1}^{2}\right\rangle$ and rewrite it in final notations.

$$
\frac{\left\langle 0\left|\prod_{j=1}^{N} C\left(\lambda_{j}\right) \mathbf{Q}_{1}^{2} \prod_{j=1}^{N} B\left(\lambda_{j}\right)\right| 0\right\rangle}{\left\langle 0\left|\prod_{j=1}^{N} C\left(\lambda_{j}\right) \prod_{j=1}^{N} B\left(\lambda_{j}\right)\right| 0\right\rangle} \equiv \frac{\left\langle\mathbf{Q}_{1}^{2}\right\rangle_{N}}{\langle\mathbf{1}\rangle_{N}}=\frac{\left\langle\mathbf{Q}_{1}^{2}\right\rangle_{N}^{0}}{\langle\mathbf{1}\rangle_{N}}+\sum_{k=2}^{N} \Gamma_{k, N} .
$$

One must remember that the s.t.e. (3.3) is valid. The $\Gamma_{k, N}$ is the contribution of the $k$ particle irreducible part

$$
\Gamma_{k, N}=\frac{\mathscr{D}_{N}^{k}}{\langle\mathbf{1}\rangle_{N}}=\sum_{\{\lambda\}=\left\{\lambda^{I}\right\} \cup\left\{\lambda^{v}\right\}} \frac{\operatorname{det}_{(N-k)}\left(\varphi_{\nu}^{\prime}\right)}{\operatorname{det}_{N}\left(\varphi^{\prime}\right)} I_{k, N}^{d}\left(\left\{\lambda^{I}\right\}_{k},\left\{\lambda^{v}\right\}_{N-k}\right) .
$$

Here we introduce a new factor $\operatorname{det}\left(\varphi_{v}^{\prime}\right)$ which will cancel the denominator of (3.37). The matrix $\varphi_{v}^{\prime}$ is defined by $\left(\varphi_{v}^{\prime}\right)_{j k}=\partial \varphi_{j}^{v} / \partial \lambda_{k}^{v}$ and by

$$
\varphi_{j}^{v}=i \ln r\left(\lambda_{j}^{v}\right)+\sum_{k \neq j} i \ln \left(\frac{f\left(\lambda_{j}^{v} \lambda_{k}^{v}\right)}{f\left(\lambda_{k}^{v} \lambda_{j}^{v}\right)}\right)
$$


Here the summation variable $\lambda_{k}^{v}$ takes all the values from the set $\left\{\lambda^{v}\right\}$ except $\lambda_{j}^{v}$. The number of values $\varphi_{j}^{v}$ is equal to the number of $\lambda_{j}^{v}$ and is equal to $N-k$. The $I_{k, N}^{d}$ is the normalized $\square_{k, N}^{d}$ :

$$
\begin{aligned}
I_{k, N}^{d}\left(\left\{\lambda^{I}\right\},\left\{\lambda^{v}\right\}\right)= & \sum_{\left\{\lambda^{I}\right\}=\left\{\lambda^{+}\right\} \cup\left\{\lambda^{-}\right\} \cup\left\{\lambda^{0}\right\}} \mathscr{A}_{k}^{n}\left(\left\{\lambda^{+}\right\},\left\{\lambda^{-}\right\},\left\{\lambda^{0}\right\}\right) \\
& \times E_{n, N-k}\left(\left\{\lambda^{+}\right\}_{n},\left\{\lambda^{-}\right\}_{n},\left\{\lambda^{v}\right\}_{N-k}\right) \prod_{j=1}^{n} \ell\left(\lambda_{j}^{+}\right) \ell^{-1}\left(\lambda_{j}^{-}\right) .
\end{aligned}
$$

Here $E_{n, N-k}$ is normalized $\mathbb{E}_{n, N-k}$ :

$$
\begin{aligned}
E_{n, N-k}\left(\left\{\lambda^{+}\right\}_{n},\left\{\lambda^{-}\right\}_{n},\left\{\lambda^{v}\right\}_{N-k}\right) & \\
& =\sum_{\left\{\lambda^{v}\right\}=\left\{\lambda^{x}\right\} \cup\left\{\lambda^{y}\right\}}\left(\frac{\operatorname{det}_{n_{x}}\left(\varphi_{x}^{\prime}\right) \operatorname{det}_{n_{y}}\left(\varphi_{y}^{\prime}\right)}{\operatorname{det}_{(N-k)}\left(\varphi_{v}^{\prime}\right)}\right) \prod_{i=1}^{n_{x}} \prod_{j=1}^{n} \frac{f\left(\lambda_{j}^{+} \lambda_{i}^{x}\right)}{f\left(\lambda_{i}^{x} \lambda_{j}^{+}\right)} \frac{f\left(\lambda_{i}^{x} \lambda_{j}^{-}\right)}{f\left(\lambda_{j}^{-} \lambda_{i}^{x}\right)} .
\end{aligned}
$$

So we have achieved our aim and expressed the correlation function in terms of the irreducible parts. It should be noted that the behaviour of $\Gamma_{k, N}$ in the strong coupling limit is the same as one of irreducible part (2.4), $\Gamma_{k, N} \rightarrow c^{2-k}$ at $c \rightarrow \infty$. So the decomposition (3.33) looks like an expression in the coupling constant. In the next sect. we shall move on to the thermodynamic limit. The order of the limits will be standard for the quantum field theory. We fix $k$ - the order in the coupling constant and tend the number of vacuum particles $(N-k)$ to infinity. In the next sect. we shall calculate the limit

$$
\lim _{N \rightarrow \infty} E_{n, N-k}\left(\left\{\lambda^{+}\right\},\left\{\lambda^{-}\right\},\left\{\lambda^{v}\right\}_{N-k}\right)=E_{n}\left(\left\{\lambda^{+}\right\}_{n},\left\{\lambda^{-}\right\}_{n}\right) .
$$

Here $n$ and $k$ are fixed. In Sect. 5 we shall calculate the limit

$$
\lim _{N \rightarrow \infty} \Gamma_{k, N}=\Gamma_{k}
$$

\section{The Dressing Equations}

Here we evaluate explicitly the limit of the function $E_{n, N}$ (3.37), (3.38). First of all let us define the thermodynamic limit for the generalized model by analogy with one of the NS model. For this purpose put $i \ln r(\lambda)=L u(\lambda)$; here $u^{\prime}(\lambda)>0$. The thermodynamic limit is $L \rightarrow \infty, N \rightarrow \infty, N / L=$ const. The momenta $\lambda_{j}$; satisfying the s.t.e. $\varphi_{j}^{\prime}=2 \pi j+\pi N,-N / 2 \leqq j \leqq N / 2$ (see (3.2), (3.3), (1.1)), fill the interval $[-q, q]$. The density $\rho_{u}\left(\lambda_{k}\right)=1 / L\left(\lambda_{k+1}-\lambda_{k}\right)$ satisfies the equation:

$$
\left[\left(\mathbf{1}-\frac{1}{2 \pi} \mathbf{K}\right) \rho_{u}\right](\mu)=u^{\prime}(\mu) / 2 \pi,
$$

see (1.3). The limit of the Jacobian is equal to

$$
\operatorname{det}_{N} \varphi^{\prime}=\prod_{j=1}^{N}\left[2 \pi L \rho_{u}\left(\lambda_{j}\right)\right] \operatorname{det}(\mathbf{1}-\mathbf{K} / 2 \pi),
$$

see Appendix A. It should be noted that in this limit the continuous function 
$x(\lambda)=i \ln ^{\prime} \ell(\lambda)$ remains fixed and finite. It is a positive function with a bounded variation. The function $y(\lambda)$ goes to infinity

$$
x_{m}>x(\lambda) \geqq 0, \quad y(u)=L u^{\prime}(\lambda)-x(\lambda) .
$$

Note. For the NS model $u(\lambda)=\lambda, u^{\prime}(\lambda)=1$.

Below we study the behavior of the function $E_{n, N}$ under the variation of function $x(\lambda)$. The function $u(\lambda)$ will be fixed, one can put $u(\lambda)=\lambda$ from the very begining.

Now we shall evaluate the limit of values $\left\langle\mathbf{Q}_{1}\right\rangle /\langle\mathbf{1}\rangle(3.23)$ and $\left\langle\mathbf{Q}_{1}^{2}\right\rangle^{0} /\langle\mathbf{1}\rangle$ (3.28). Let us introduce the generating function:

$$
E_{N}(\alpha,[x(\lambda)])=\sum_{\{\lambda\}=\left\{\lambda^{x}\right\} \cup\left\{\lambda^{3}\right\}} e^{\alpha n_{x}} \frac{\operatorname{det}_{n_{x}}\left(\varphi_{x}^{\prime}\right) \operatorname{det}_{n_{y}}\left(\varphi_{y}^{\prime}\right)}{\operatorname{det}_{N}\left(\varphi^{\prime}\right)} .
$$

We have written down explicitly the functional argument $x(\lambda)$. Due to (3.18), (3.23) and (3.28) we have:

$$
\left.E_{N}\right|_{\alpha=0}=1,\left.\frac{\partial}{\partial \alpha} E_{N}\right|_{\alpha=0}=\frac{\left\langle\mathbf{Q}_{1}\right\rangle_{N}}{\langle\mathbf{1}\rangle_{N}},\left.\frac{\partial^{2}}{\partial \alpha^{2}} E_{N}\right|_{\alpha=0}=\frac{\left\langle\mathbf{Q}_{1}^{2}\right\rangle_{N}^{0}}{\langle\mathbf{1}\rangle_{N}} .
$$

We put $\operatorname{Re} \alpha=0$. The first property of $E$ is

$$
E_{N}(\alpha,[x(\lambda)])=1 \quad \text { at } \quad x(\lambda)=0,
$$

see (3.16). Then we prove the following

Theorem 4. The modulus of the function $E_{N}$ is less than one:

$$
\mid E_{N}(\alpha,[x(\lambda)] \mid \leqq 1
$$

Proof. The positiveness of the determinants $\operatorname{det} \varphi^{\prime}>0, \operatorname{det} \varphi_{x}^{\prime}>0, \operatorname{det} \varphi_{y}^{\prime}>0$, which is proved in Appendix $\mathrm{B}$, permits us to make the following estimation: $\left|E_{N}(\alpha,[x])\right| \leqq E_{N}(0,[x]), E_{N}(0,[x])=1$, see (4.4), (4.5).

This completes the proof. Let us consider the thermodynamic limit of $E_{N}$.

Theorem 5. The thermodynamic limit of $E_{N}(\alpha,[x])$ is equal to

$$
E(\alpha,[x(\lambda)])=\exp \left\{\int_{-q}^{q} x(t) P(t, \alpha) d t\right\} .
$$

Here function $P(t, \alpha)$ is defined at $-q \leqq t \leqq q$ and $\operatorname{Re} \alpha=0$. This function is defined in the unique way by the nonlinear integral equation

$$
2 \pi P(t, \alpha)=\exp \left\{\alpha+\int_{-q}^{q} K(t, s) P(s, \alpha) d s\right\}-1
$$

and by inequality $\operatorname{Re} P(t, \alpha) \leqq 0$.

Proof. Let us separate $E_{N}$ into two parts

$$
E_{N}(\alpha,[x])=E_{N}^{(1)}(\alpha,[x])+E_{N}^{(2)}(\alpha,[x]),
$$


with

$$
E_{N}^{(1)}(\alpha,[x])=\sum_{\{\lambda\}=\left\{\lambda^{x}\right\} \cup\{\lambda y\}}^{n_{x} \leqq \ln N} e^{\alpha n_{x}} \frac{\operatorname{det}_{n_{x}}}{\operatorname{det}_{N}\left(\varphi_{x}^{\prime}\right)} \frac{\operatorname{det}_{n_{y}}}{\left.\operatorname{lot}^{\prime}\right)} \underline{\left(\varphi_{y}^{\prime}\right)}
$$

The value $E_{N}^{(2)}$ is the complement of $E_{N}^{(1)}$ with respect to the whole $E_{N}$.

Remark. The properties (4.6), (4.7) are valid for $E_{N}^{(1)}$ also.

Let us transform the expression for $E_{N}^{(1)}$. The values $N$ and $n_{y}$ go to infinity, so the two determinants can be simplified, like (4.2). The explicit formulae are presented in Appendix A. The asymptotical expression is:

$$
E^{(1)}=\sum_{\{\lambda\}=\left\{\lambda^{x}\right\} \cup\{\lambda y\}}^{n_{x} \leqq \ln N} \exp \left\{\alpha n_{x}-\int_{-q}^{q} x(\lambda) \frac{d \lambda}{2 \pi}\right\} \operatorname{det}_{n_{x}}\left(\varphi_{x}^{\prime}\right) \prod_{j=1}^{n_{x}}\left[\frac{\omega\left(\lambda_{j}^{x}\right)}{2 \pi L \rho_{u}\left(\lambda_{j}^{x}\right)}\right]
$$

for $\omega$, see (2.13). Let us evaluate the contribution of the terms with fixed $n_{x}$. In the limit it will be a $n_{x}$-multiple integral, see (1.4):

$$
W\left(n_{x}\right)=\left(\frac{\exp \left\{\alpha n_{x}-\int_{-q}^{q} \frac{x(\lambda) d \lambda}{2 \pi}\right\}}{\left(n_{x}\right) !}\right) \int_{-q}^{q} \operatorname{det}_{n_{x}}\left(\varphi_{x}^{\prime}\right) \prod_{j=1}^{n_{x}}\left(\frac{\omega\left(\lambda_{j}^{x}\right)}{2 \pi} d \lambda_{j}^{x}\right) .
$$

So we have $E_{N}^{(1)}=\sum_{n_{x}=0}^{[\ln N]} W\left(n_{x}\right)$. It is proved in Appendix $\mathrm{C}$ that the limit of this sum at $N \rightarrow \infty$ exists and is equal to

$$
E(\alpha,[x(\lambda)])=\sum_{n=0}^{\infty} W(n), \quad W(0)=\exp \left\{-\int_{-q}^{q} \frac{x(\lambda) d \lambda}{2 \pi}\right\}
$$

Derive now a linear equation in variational derivatives for this functional. Let us vary it with respect to the function $x(\mu)$. The answer is the following:

$$
\frac{\delta E(\alpha,[x(\lambda)])}{\delta x(\mu)}=\frac{-1}{2 \pi} E(\alpha,[x(\lambda)])+\left(\frac{e^{\alpha}}{2 \pi}\right) E(\alpha,[x(\lambda)+K(\lambda, \mu)]) .
$$

Here we use the property of the determinant (3.14), (3.15). One can obtain all the solutions of the linear differential equation by means of the Fourier transformation

$$
E(\alpha,[x(\mu)])=\exp \left\{\int_{-q}^{q} x(t) P(t, \alpha) d t\right\},
$$

see (4.6). From (4.15) we obtain

$$
2 \pi P(t, \alpha)=\exp \left\{\alpha+\int_{-q}^{q} K(t, s) P(s, d) d s\right\}-1,
$$

and from (4.7) $\operatorname{Re} P(t, \alpha) \leqq 0$. In Appendix D the uniqueness theorem is proved for this system. So we have calculated the limit of $E_{N}^{(1)}$ (4.11). In Appendix C it is proved that the limit of $E_{N}^{(2)}$ in (4.10) is equal to zero. This completes the proof. Now we are able to calculate the mean values (4.5)

$$
\left\langle\mathbf{Q}_{1}\right\rangle /\langle\mathbf{1}\rangle=\partial E /\left.\partial \alpha\right|_{\alpha=0}=\int x(t) P^{\prime}(t) d t
$$


Here $P^{\prime}(t)$ is defined by the equation $\left[(2 \pi-\mathbf{K}) P^{\prime}\right]=1$, see (1.3). Comparing with (1.2) we see that $P^{\prime}(t)=\rho(t)$. Note that for the NS model $\left\langle\mathbf{Q}_{1}\right\rangle /\langle\mathbf{1}\rangle=x \int \rho(\lambda) d \lambda$. For $\left\langle\mathbf{Q}_{1}^{2}\right\rangle^{0}$, we have:

$$
\frac{\left\langle\mathbf{Q}_{1}^{2}\right\rangle^{0}}{\langle\mathbf{1}\rangle}=\left.E^{\prime \prime}\right|_{\alpha=0}=\left[\int_{-q}^{q} x(t) P^{\prime}(t) d t\right]^{2}+\int_{-q}^{q} x(t) P^{\prime \prime}(t) d t
$$

Here $P^{\prime \prime}(t)$ is defined by the integral equation $\left[(2 \pi-\mathbf{K}) P^{\prime \prime}\right]=\left(2 \pi P^{\prime}\right)^{2}$. Now let us calculate the thermodynamic limit of $E_{n, N-k}(3.37),(3.38)$.

Theorem 6. The limit of $E_{n, N-k}\left(\left\{\lambda^{+}\right\},\left\{\lambda^{-}\right\},\left\{\lambda^{v}\right\}\right)$ at $N \rightarrow \infty ; n$, k-fixed is equal to

$$
E_{n}\left(\left\{\lambda^{+}\right\},\left\{\lambda^{-}\right\}\right)=\exp \left\{\int_{-q}^{q} x(t) P_{n}\left(t,\left\{\lambda^{+}\right\},\left\{\lambda^{-}\right\}\right) d t\right\} \text {. }
$$

The function $P_{n}$ is defined in the unique way be the equation:

$$
2 \pi P_{n}(t)=\left[\prod_{j=1}^{n} \frac{f\left(\lambda_{j}^{+} t\right)}{f\left(t, \lambda_{j}^{+}\right)} \frac{f\left(t, \lambda_{j}^{-}\right)}{f\left(\lambda_{j}^{-} t\right)}\right] \exp \left\{\int_{-q}^{q} K(t, s) P_{n}(s) d s\right\}-1,
$$

and by inequality $\operatorname{Re} P_{n} \leqq 0$. Here $-q \leqq t \leqq q,-q \leqq \lambda_{j}^{ \pm} \leqq q$.

The proof is similar to that of Theorems 4,5 . So we have evaluated the thermodynamic limit of $E_{n, N}$. It will help us to calculate the thermodynamic limit of $\Gamma_{k, N}$ in the next sect.

\section{The Contribution of the $k$-Particle Processes to the Correlation Function}

Here we calculate the limit of $\Gamma_{k, N}(3.34),(3.39)$. The limit of $I_{k, N}^{d}$ is equal to

$$
I_{k}^{d}\left(\left\{\lambda^{I}\right\}_{k}\right)=\sum_{\left\{\lambda^{I}\right\}=\left\{\lambda^{+}\right\}_{n} \cup\left\{\lambda^{-}\right\}_{n} \cup\left\{\lambda^{0}\right\}} \mathscr{A}_{k}^{n}\left(\left\{\lambda^{+}\right\},\left\{\lambda^{-}\right\},\left\{\lambda^{0}\right\}\right) E_{n}\left(\left\{\lambda^{+}\right\},\left\{\lambda^{-}\right\}\right) \prod_{j=1}^{n} \ell\left(\lambda_{j}^{+}\right) \ell^{-1}\left(\lambda_{j}^{-}\right),
$$

see (3.36), (4.19). The calculations similar to those of Appendix A show that $\operatorname{det}_{N-k}\left(\varphi_{v}^{\prime}\right) / \operatorname{det}_{N} \varphi^{\prime}=\prod_{j=1}^{k}\left[\omega\left(\lambda_{j}^{I}\right) / 2 \pi L \rho_{u}\left(\lambda_{j}^{I}\right)\right]$. For $\Gamma_{k, N}$ we obtain the asymptotical expression $(N \rightarrow \infty)$ :

$$
\Gamma_{k, N}=\sum_{\{\lambda\}_{N}=\left\{\lambda^{I}\right\}_{k} \cup\left\{\lambda^{\nu}\right\}_{N-k}} I_{k}^{d}\left(\left\{\lambda^{I}\right\}\right) \prod_{j=1}^{k}\left[\frac{\omega\left(\lambda_{j}^{I}\right)}{2 \pi L \rho_{u}\left(\lambda_{j}^{I}\right)}\right] .
$$

As the $I_{k}^{d}\left(\left\{\lambda^{I}\right\}\right)$ is a symmetric, bounded function of all $\lambda_{j}^{I}$ we can replace the sum by an integral as was done in (1.4). Finally the limit of $\left(N \rightarrow \infty, k\right.$-fixed) $\Gamma_{k, N}$ is equal to a $k$-multiple integral:

$$
\Gamma_{\bullet k}=\frac{1}{k !} \int_{-q}^{q} \prod_{j=1}^{k}\left[\frac{\omega\left(\lambda_{j}\right) \mathrm{d} \lambda_{j}}{2 \pi}\right] \mathrm{I}_{k}^{d}(\{\lambda\})
$$

see (2.13). For the mean value of $\mathbf{Q}_{1}^{2}$ with respect to the physical vacuum, we have 
(4.18), (3.33), (1.2):

$$
\begin{gathered}
\frac{\left\langle\Omega\left|\mathbf{Q}_{1}^{2}\right| \Omega\right\rangle}{\langle\Omega \mid \Omega\rangle}=\left[\int_{-q}^{q} x(\lambda) P^{\prime}(\lambda) d \lambda\right]^{2}+\int_{-q}^{q} x(\lambda) P^{\prime \prime}(\lambda) d \lambda+\sum_{k=2}^{\infty} \Gamma_{k} ; \\
P^{\prime}(\lambda)=\rho(\lambda) .
\end{gathered}
$$

So one can see that we can explicitly evaluate the dependence on the arbitrary function $\ell(\lambda)$ in the generalized model. We can not however explicitly evaluate the dependence of the correlation function on the $R$ matrix as we have no simple formula for the Fourier coefficients $\mathscr{A}_{n}^{k}$. These coefficients must be calculated step by step, using the methods of paper [1]. Now let us pass to the NS model. For it, $\ell(\lambda)=\exp \{-i \lambda x\}, x(\lambda)=x$. In this case formula (5.4) looks like

$$
\frac{\left\langle\Omega\left|\mathbf{Q}_{1}^{2}\right| \Omega\right\rangle}{\langle\Omega \mid \Omega\rangle}=x^{2}\left[\int_{-q}^{q} \rho(\lambda) d \lambda\right]^{2}+x \int_{-q}^{q} P^{\prime \prime}(\lambda) d \lambda+\sum_{k=2}^{\infty} \Gamma_{k} .
$$

In the expression for $\Gamma_{k}$ one must put into (5.1) that

$$
E_{n} \prod_{j=1}^{n} \ell\left(\lambda_{j}^{+}\right) \ell^{-1}\left(\lambda_{j}^{-}\right)=\exp \left\{-i x \sum_{j=1}^{n}\left(\lambda_{j}^{+}-\lambda_{j}^{-}\right)+x \int_{-q}^{q} P_{n}\left(t,\left\{\lambda^{+}\right\},\left\{\lambda^{-}\right\}\right) d t\right\} .
$$

To obtain the correlation function of the currents one must use formula (2.1). This completes the proof of formula (2.15).

\section{Conclusions}

We have constructed the consistent perturbation theory for correlation functions of the currents for the NS model. Our approach is quite general. The increase of the number of sites in the generalized model gives us the opportunity to calculate any correlation function. As it was mentioned in [1] one needs a four-site generalized model to calculate the correlation functions of the fields $\left\langle\psi(x) \psi^{+}(y)\right\rangle$. Let us describe the answer. Any correlation function is equal to a mean value of some operator $\mathbf{o}$ with respect to the physical vacuum $\langle\Omega|\mathbf{o}| \Omega\rangle$. To construct the decomposition of this correlation function one must calculate the mean value of the operator $\mathbf{o}$ with respect to the $k$ particle eigenstate of the Hamiltonian $\left\langle\Psi_{k}|\mathbf{o}| \Psi_{k}\right\rangle$, then take its irreducible part (zero coefficient in (2.2)), dress it by means of equations similar to (2.8), and then integrate. For the correlation function of the currents this decomposition gives the improved version of the $1 / c$ expansion. However, generally speaking, for an arbitrary correlation function all terms of the series are essential. For example, the correlation function $\left\langle\psi(x) \psi^{+}(y)\right\rangle$ can be expressed as a series at any $0<c<\infty$ by means of our method. However, all terms of this series for $\left\langle\psi(x) \psi^{+}(y)\right\rangle$ are essential at $c \rightarrow \infty$, and not only the first term as for current correlator $\langle j(x) j(y)\rangle$. Our approach can be applied to any model with the $R$ matrix of the XXX or XXZ models. This set includes XXZ Heisenberg model and the sineGordon model. Note, for example, that the XXX Heisenberg model and the NS model are special cases of the same generalized model [1]. The decomposition (5.4) is natural from the point of view of the analogy of the quantum inverse scattering 
method and the theory of groups representations. Indeed, the "representation" is parametrized by the function $a(\lambda) / d(\lambda)$ [18]. We succeeded in evaluation of the dependence of the correlation function on this arbitrary function (the coefficients $\mathscr{A}_{k}^{n}$ depend only on the $R$-matrix).

Let us discuss the series (2.15) and make some hypothesis. The series seems to be convergent due to the weight $\omega(\lambda)$. Its contribution can be estimated as $\omega_{0}^{k}(2.14)$ $\left(0<\omega_{0}<1\right)$. All other factors in expression (2.12) depend on $k$ in a polynomial way. The analysis of the series leads to the following estimation:

$$
0 \leqq \frac{\left\langle\Omega\left|: j\left(x_{1}\right) j\left(x_{2}\right):\right| \Omega\right\rangle}{\langle\Omega \mid \Omega\rangle} \leqq\left[\int_{-q}^{q} \rho(\lambda) d \lambda\right]^{2}
$$

\section{Appendix A}

Let us consider the Jacobian $\operatorname{det} \varphi^{\prime}$ in the generalized model. We write the matrix (see the beginning of Sect. 3)

$$
\varphi_{j \ell}^{\prime}=\delta_{j \ell}\left[z_{j}+\sum_{m=1}^{N} K_{j m}\right]-K_{j \ell}
$$

as a product of two factors:

$$
\varphi^{\prime}=\mathbf{G} \Theta, \quad \operatorname{det} \varphi^{\prime}=\operatorname{det} \mathbf{G} \operatorname{det} \Theta .
$$

Here

$$
\Theta_{j \ell}=\delta_{j \ell} \vartheta_{\ell}, \quad \vartheta_{\ell}=z_{\ell}+\sum_{m=1}^{N} K_{\ell m}>0
$$

and

$$
G_{j \ell}=\delta_{j \ell}-K_{j \ell} / \vartheta_{\ell} .
$$

The representations similar to (A.1)-(A.4) are valid for matrices $\varphi_{x}^{\prime}$ and $\varphi_{y}^{\prime}$. They can be obtained from (A.1)-(A.4) by replacement of $z_{j}$ by $x_{j}$ or by $y_{j}$ respectively. The thermodynamic limit (see the beginning of Sect. 4) of $\vartheta_{\ell}$ is equal to

$$
\vartheta_{\ell}=2 \pi L \rho_{u}\left(\lambda_{\ell}\right)
$$

Here we replaced $\Sigma$ by an integral like in (1.4), and use the equation (4.1). The determinant of $\Theta$ is equal to $\operatorname{det} \Theta=\prod_{j=1}^{N}\left[2 \pi L \rho_{u}\left(\lambda_{j}\right)\right]$. The operator $\mathbf{G}$ in the limit turns into the integral operator (1.2), (1.3) $\mathbf{G}=(\mathbf{1}-\mathbf{K} / 2 \pi)$. So the final answer is

$$
\operatorname{det}_{N} \varphi^{\prime}=\left\{\prod_{j=1}^{N}\left[2 \pi L \rho_{u}\left(\lambda_{j}\right)\right]\right\} \operatorname{det}(\mathbf{1}-\mathbf{K} / 2 \pi) .
$$

Now let us consider $\operatorname{det}_{n_{y}}\left(\varphi_{y}^{\prime}\right)$ from (4.11). Here $n_{y} \geqq(N-\ln N)$ goes to infinity in the thermodynamic limit. We represent the matrix $\varphi_{y}^{\prime}$ in the form (A.2) $\varphi_{y}^{\prime}=\mathbf{G}^{y} \Theta^{y}$ with

$$
\begin{gathered}
\Theta_{j \ell}^{y}=\delta_{j \ell} \vartheta_{\ell}^{y} ; \quad \vartheta_{\ell}^{y}=y\left(\lambda_{\ell}^{y}\right)+\sum_{m=1}^{n_{y}} K\left(\lambda_{\ell}^{y}, \lambda_{m}^{y}\right), \\
G_{j \ell}^{y}=\delta_{j \ell}-K\left(\lambda_{j}^{y} \lambda_{\ell}^{y}\right) / \vartheta_{\ell}^{y} .
\end{gathered}
$$


By means of (3.8), (3.9), (4.3) and (A.5) one can calculate the limit of $\vartheta_{\ell}^{y}$ :

$$
\vartheta_{j}^{y}=2 \pi L \rho_{u}\left(\lambda_{j}^{y}\right)-x\left(\lambda_{j}^{y}\right)-\sum_{\ell=1}^{n_{x}} K\left(\lambda_{j}^{y} \lambda_{\ell}^{x}\right) .
$$

The determinant of $\Theta^{y}$ is equal to

$$
\begin{gathered}
\frac{\operatorname{det} \Theta^{y}}{\prod_{m=1}^{n_{y}}\left[2 \pi L \rho_{u}\left(\lambda_{m}^{y}\right)\right]}=\prod_{j=1}^{n_{y}}\left[1-\frac{x\left(\lambda_{j}^{y}\right)+\sum_{\ell=1}^{n_{x}} K\left(\lambda_{j}^{y} \lambda_{\ell}^{x}\right)}{2 \pi L \rho_{u}\left(\lambda_{j}^{y}\right)}\right] \\
\rightarrow \exp \left\{\frac{-1}{2 \pi} \int_{-q}^{q}\left[x(\mu)+\sum_{\ell=1}^{n_{x}} K\left(\mu, \lambda_{\ell}^{x}\right)\right] d \mu\right\},
\end{gathered}
$$

see (1.4). Here the sum is over the set $\left\{\lambda^{x}\right\}$ (3.9). In (A.9) the weight $\omega(\lambda)(2.13)$ appears. The limit of $\mathbf{G}^{y}$ is exactly the same as that of $\mathbf{G}, \mathbf{G}^{y} \rightarrow(\mathbf{1}-\mathbf{K} / 2 \pi)$. So the asymptotical expression for the Jacobian is

$$
\frac{\operatorname{det}_{n_{y}}\left(\varphi_{y}^{\prime}\right)}{\operatorname{det}(\mathbf{1}-\mathbf{K} / 2 \pi)}=\left\{\prod_{j=1}^{n_{y}}\left[2 \pi L \rho_{u}\left(\lambda_{j}^{y}\right)\right]\right\}\left\{\prod_{\ell=1}^{n_{x}} \omega\left(\lambda_{\ell}^{x}\right)\right\} \exp \left\{-\int_{-q}^{q} \frac{x(\lambda) d \lambda}{2 \pi}\right\} .
$$

Note. The asymptotical expression for $\operatorname{det} \varphi_{v}^{\prime}(3.35)$ is similar, but the last factor is absent.

The ratio of two determinants (A.6) and (A.10) is equal to

$$
\frac{\operatorname{det}_{n_{y}}\left(\varphi_{y}^{\prime}\right)}{\operatorname{det}_{N}\left(\varphi^{\prime}\right)}=\left\{\prod_{\ell=1}^{n_{x}}\left[\frac{\omega\left(\lambda_{\ell}^{x}\right)}{2 \pi L \rho_{u}\left(\lambda_{\ell}^{x}\right)}\right]\right\} \exp \left\{\frac{-1}{2 \pi} \int_{-q}^{q} x(\lambda) d \lambda\right\}
$$

\section{Appendix B}

Let us prove that $\varphi^{\prime}>0$ at $z_{j}>0$. If $v_{j}$ is a real vector, then the quadratic form

$$
\sum_{i, j=1}^{N} \varphi_{i j}^{\prime} v_{i} v_{j}=\sum_{j=1}^{N} z_{j} v_{j}^{2}+\sum_{j>i} K_{j i}\left(v_{j}-v_{i}\right)^{2}
$$

is positive, see (A.1). It means that

$$
\varphi^{\prime}>0, \quad \operatorname{det} \varphi^{\prime}>0 .
$$

It leads to $1>\mathbf{G}>0$, see (A.2)-(A.4). In the thermodynamical limit the operator

$$
1>1-\mathbf{K} / 2 \pi>0
$$

is positive and nondegenerate, see [5-7].

\section{Appendix C}

The value $E_{N}^{(1)}(0,[x])$ is less than $E_{N}(0,[x])$, see (4.4), (4.11) at $\alpha=0$ :

$$
E_{N}^{(1)}(0,[x])<E_{N}(0,[x])=1 .
$$

Here we use (4.5) and (B.2). In the thermodynamical limit it means that

$$
E_{N}^{(1)}(0,[x])=\left.\sum_{n_{x}=0}^{[\ln N]} W\left(n_{x}\right)\right|_{\alpha=0} \leqq 1 .
$$


As $W(n)>0$ at $\alpha=0, E_{N}^{(1)}$ form monotonically increasing, bounded sequence. So at $N \rightarrow \infty$ this sequence has a limit, and the series

$$
\left.\sum_{n=0}^{\infty} W(n)\right|_{\alpha=0}
$$

is covergent. It means that the series (4.14) is absolutely convergent as $|W(n)|=W(n)$ at $\alpha=0$.

Now let us prove that the limit of $E_{N}^{(2)}(\alpha)$ in (4.10) is equal to zero. The standard estimation (see (B.2)) shows that

$$
\left|E_{N}^{(2)}(\alpha,[x])\right| \leqq E_{N}^{(2)}(0,[x]) .
$$

By means of (4.5), (4.10) one has:

$$
E_{N}^{(2)}(0,[x])=1-E_{N}^{(1)}(0,[x]) .
$$

Using Eq. (4.16) and the fact that Eq. (4.17) at $\alpha=0$ has only one solution $P(t, 0)=0$, we obtain

$$
E_{N}^{(1)}(0,[x]) \underset{N \rightarrow \infty}{\longrightarrow} 1
$$

It means that

$$
\lim _{N \rightarrow \infty} E_{N}^{(2)}(0,[x])=\lim _{N \rightarrow \infty} E_{N}^{(2)}(\alpha,[x])=0
$$

\section{Appendix D}

Here we prove the uniqueness theorem for Eq. (4.17) or (4.9). Let us suppose that we have two solutions $P_{(1)}(t)$ and $P_{(2)}(t): \operatorname{Re} P_{(1,2)} \leqq 0$,

$$
2 \pi P_{(1)}-\exp \left\{\alpha+\mathbf{K} P_{(1)}\right\}=-1 ; 2 \pi P_{(2)}-\exp \left\{\alpha+\mathbf{K} P_{(2)}\right\}=-1,
$$

see (1.3). Let us subtract the equations and prove the estimate

$$
0=\mid 2 \pi\left(P_{(1)}-P_{(2)}\right)-e^{\alpha}\left(e^{\mathbf{K} P_{(1)}}-e^{\left.\mathbf{K} P_{(2)}\right)}|\geqq(2 \pi-\mathbf{K})| P_{(1)}-P_{(2)} \mid \geqq 0 .\right.
$$

It will prove the theorem as the equation $(2 \pi-\mathbf{K})\left|P_{(1)}-P_{(2)}\right|=0$ has only one solution $P_{(1)}=P_{(2)}$, see Appendix B. The first step in the proof of the estimate is:

$$
\mid 2 \pi\left(P_{(1)}-P_{(2)}\right)-e^{\alpha}\left(e^{\mathbf{K} P_{(1)}}-e^{\left.\mathbf{K} P_{(2)}\right)}|\geqq 2 \pi| P_{(1)}-P_{(2)}|-| e^{\mathbf{K} P_{(1)}}-e^{\mathbf{K} P_{(2)} \mid} .\right.
$$

As $\operatorname{Re} K P_{(1.2)} \leqq 0(\mathbf{K}$ is a positive, see (1.3)) we have

$$
\left|\exp \left\{\mathbf{K} P_{(1)}\right\}-\exp \left\{\mathbf{K} P_{(2)}\right\}\right| \leqq\left|\mathbf{K} P_{(1)}-\mathbf{K} P_{(2)}\right| \leqq \mathbf{K}\left|P_{(1)}-P_{(2)}\right| .
$$

It proves the estimate and concludes the proof of the uniqueness theorem.

Acknowledgements. I thank L. D. Faddeev, A. G. Izergin, F. A. Smirnov and A. N. Kirilov for discussions.

\section{References}

1. Izergin, A. G., Korepin, V. E.: The quantum inverse scattering method approach to the correlation functions. Commun. Math. Phys. 93, - (1984)

2. Faddeev, L. D.: Quantum completely integrable models of field theory. Sov. Sci. Rev. Math. Phys. C1, 107-160 (1981) 
3. Faddeev, L. D., Sklyanin, E. K.: Quantum mechanical approach to the completely integrable models of field theory. Dokl. Akad. Nauk SSSR 243, 1430-1433 (1978)

4. Sklyanin, E. K.: The inverse scattering method and the quantum nonlinear Schrödinger equation. Dokl. Akad. Nauk SSSR 244, 1337-1341 (1978)

5. Lieb, E. H.: Exact Analysis of an Interacting Bose Gas I. The General Solution and the Ground State. Phys. Rev. 130, 1605-1616 (1963)

6. Lieb, E. H.: Exact Analysis of an Interacting Bose Gas II. The Excitation Spectrum. Phys. Rev. 130, $1616-1624$ (1963)

7. Yang, C. N., Yang, C. P.: Thermodynamics of a One-Dimensional System of Bosons with Repulsive Delta-Function Interaction. Journ. Math. Phys. 10, 1115-1122 (1969)

8. Korepin, V. E.: A Direct calculation of the $S$-matrix of the Massive Thirring Model. Teor. Mat. Phys. 41, 169-189 (1979)

9. Korepin, V. E., Calculation of Norms of Bethe Wave Functions. Commun. Math. Phys. 86, 391-418 (1982)

10. Destri, C., Lowenstein, J. H.: Normalization of Bethe-Ansatz States in the Chiral-Invariant GrossNeveu Model. Preprint New-York University, NYU/TR6/82, (1-19) (1982)

11. Lenard, A.: Momentum Distribution in the Ground State of the One-Dimensional System of Impenetrable Bosons. J. Math. Phys. 5, 930-943 (1964)

12. Lenard, A.: One-Dimensional Impenetrable Boson in Thermal Equilibrium. J. Math. Phys. 7, 12681272 (1966)

13. Jimbo, M., Miwa, T., Mori, Y., Sato, M.: Density Matrix of an Impenetrable Gas and the Fifth Painleve Transcendent. Physica D1, 80-158 (1980)

14. Creamer, O. B., Thacker, H. B., Wilkinson, D.: Some Exact Results for the Two Point Function of an Integrable Quantum Field Theory. Phys. Rev. D21, 1523-1535 (1980)

15. Thacker, H. B.: The Quantum Inverse Method and Green's Functions for Completely Integrable Field Theories. Preprint. Fermilab-Conf-81/47-THY (1981)

16. Popov, V. N.: Longwave Asymptotics of the Manyparticle Green Functions for the OneDimensional Bose Gas. Pisma ZETPh 31, 560-563 (1980)

17. Karowski, M.: The Bootstrap Program for $1+1$ Dimensional Field Theoretic Models With Soliton Behaviour. In: Field Theoretical Methods in Particle Physics. Ruhl, W. (ed.). New York: Plenum Publishing Corporation 1980

18. Korepin, V. E.: Analysis of the bilinear relation of the six vertex model. Doklady AN SSSR 265, 13611364 (1982)

Communicated by A. Jaffe

Received August 31, 1983; in revised form December 8, 1983 
